\title{
Research on $U$ type gantry crane structure parametric finite element analysis system based on C\# and APDL
}

\author{
Gening Xu, Yanfei Tao* and Wenju Liu \\ School of Machinery Engineering, Taiyuan University of Science and Technology, \\ Taiyuan 030024,China \\ E-mail:475865760@qq.com
}

Key words: gantry crane, C\#, APDL programming technology, parametric modeling, finite element. Abstract: Aiming at the modeling of finite element software is applied to product design in the analysis of complex, heavy workload, time-consuming problem, proposed programming language using C\# language and ANSYS provides two APDL development tools for the overall structure parameters of type $U$ gantry crane as the object, using the APDL language to establish the gantry crane parametric finite element analysis model, combined with $\mathrm{C \#} \mathrm{development} \mathrm{a} \mathrm{set} \mathrm{of} \mathrm{parametric}$ design interface analysis system, through the system calculation compared with the theoretical calculation results, the maximum stress difference of middle span is $1.48 \%$, the maximum deformation difference is $1.2 \%$; the maximum stress difference of end span is $4.7 \%$, and the maximum deformation difference is $6 \%$, indicates that the system provides accurate and reliable calculation, provide analysis has engineering significance of gantry crane.

\section{Introduction}

With the development of CAD/CAE technology, the main method in the design of gantry crane is the traditional calculating formula and empirical estimation, the gantry crane design the same shape of different types often need to do a lot of repeated or similar work, the establishment of finite element on the need to spend a lot of time and energy analysis model, which not only reduces the design efficiency, and is easy to make a mistake. It is necessary to improve the efficiency of finite element analysis to promote the upgrading of the crane. But in the actual design process, on the one hand because of the diversity of gantry crane structure complexity and the conditions of establishing the finite element model for gantry crane is usually required for the actual structure is simplified; on the other hand, the application of ANSYS finite element analysis, the GUI operation is relatively complex, and complicated of process modeling. Therefore, it is necessary to develop a model to simplify the design of gantry crane is accurate, friendly interface and simple operation analysis system, and improve the design efficiency of gantry crane, introduced method of parametric product design.

This paper takes the U type gantry crane as the research object, using ANSYS finite element analysis system in APDL language, describes the development of design calculation model and how to create mechanical properties the parameters and the use of C\# and APDL language under the.NET platform, the parametric modeling process, and finally through the comparison with the result of theoretical analysis to verify the feasibility of the parametric modeling method. In order to achieve a series of products with the same structure, standardized analysis, making the complex finite element before and after processing, become simple and easy to use $\mathrm{e}^{[1]}$.

\section{software and language development}

ANSYS software is the main FEA program ${ }^{[2]}$ secondary development tools provided by ANSYS can be divided into three types: parametric design language (APDL), the user interface design language (UIDL) and user programmable features (UPFS) ${ }^{[3]}$.

$\mathrm{C \#}$ is a programming language of object oriented strategy for.NET Microsoft Corp specially developed, it draws on the many advantages of VB, C++ and JAVA, which can develop a Windows 
desktop application, and can develop Web application page. It uses a Visual Studio DE (integrated development environment) in the corresponding development tools Visual C\# development, use DE to create a program that can greatly shorten the time required, with strong efficiency ${ }^{[4]}$. The DE version of this procedure is 2012, the corresponding.NET version is 4.5.

APDL is the abbreviation of ANSYS Parametric Design Language, namely ANSYS parametric design language, it is an interpreted language similar to FORTRAN, to provide a general programming language features, such as parameter, macro, scalar, vector and matrix operations, a branch of the cycle, repeat and access ANSYS finite element database, it also provides a simple interface to customize the function and achieve to parameters interactive input, message mechanism, interface and operation procedures using drive $\mathrm{e}^{[5]}$.

\section{Method of parametric modeling and process planning}

\section{Method of parametric modeling}

Parametric modeling is a way to rapidly construct and modify the product modeling by using the structure of geometry feature data, which is one of the main means to realize the design and analysis of automation. It applies to the same basic structure ,but the characteristic parameters require variable series product modeling and analysis.

\section{The process of parametric modeling}

The flow chart of parametric modeling is shown in Fig. 1. Firstly, the parameters of the crane are input in the parametric modeling program, and then run the program to generate the APDL file, the generated ANSYS file by the program control APDL call, After the analysis, the model and data files needed by the users and the stress and deformation of the post process are obtained. Comparison of data which obtained by transport analysis and theory calculated.

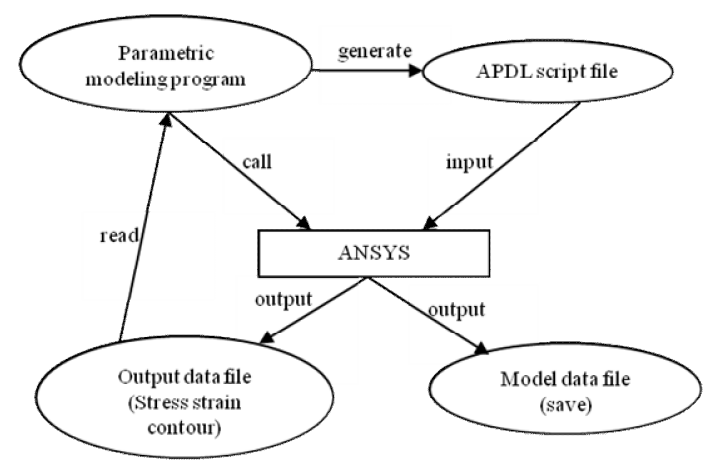

Fig. 1

\section{Development platform and implementation of parametric finite element analysis system}

\section{Development platform}

The desktop platform of the finite element parametric modeling and analysis software of gantry crane is developed using WPF. WPF is the abbreviation of Windows Presentation Foundation, its design language is XAML, which is extensible application markup language. The language has a strong ability to expand, using parameter driven controls, In order to control the location of the control, It uses the Grid mesh and a variety of Panel attribute positioning.

The modeling process of APDL command flow and GUI (graphical user interface) in different ways, the parametric modeling of the command flow mode is more emphasis on the order. In this paper, the model adopts shell element SHELL63, the finite element model is constructed from the bottom up method. Where the values of the defined dimensions are required with a parameter variable name instead of or use of other dimensions associated with it, which can achieve the effect of parameterization. When defining the key point of modeling, according to the relationship between the points in the coordinate system determine the Coordinate values ${ }^{[6]}$.

\section{Selection of parameters}

The thought of parametric finite element analysis is based on the features of structure design and analysis of specific requirements, use the parameters describing the feature size, and when establishing finite element model and analysis, using parameter table to process, so as to realize the parameter variable structure finite element analysis. The parameters of the gantry crane which 
are discussed in this paper include the dimension parameter, the material characteristic parameter, the form parameter. The size parameters including span, main girder height, flange plate thickness; Material properties parameters include elastic modulus, Poisson's ratio, density etc. The formal parameters are used to determine the fixed track, constraint etc.

\section{Parametric finite element analysis process}

ANSYS provides two entity modeling approaches: bottom-up modeling and top-down modeling. For gantry crane is discussed in this paper, using the bottom-up approach to establish the finite element model, the advantage is that parameters can be set through the program, it can easily calculate the key point about the number of any program, and can also be well controlled by the key points to establish a solid number of decent numbers, so that it can provide convenience for the solid surface with different real constant when the real constant operation is set up. In addition, the application of the load, the constraint, the extraction of support and the design parameters of the various design parameters are also easier to operate, the analysis process is shown in Fig. 2.

\section{The establishment of parametric finite element analysis system \\ Using Visual Studio 2012 as the development platform, and APDL language of ANSYS as a tool to achieve} parametric finite element analysis of the gantry crane. The system

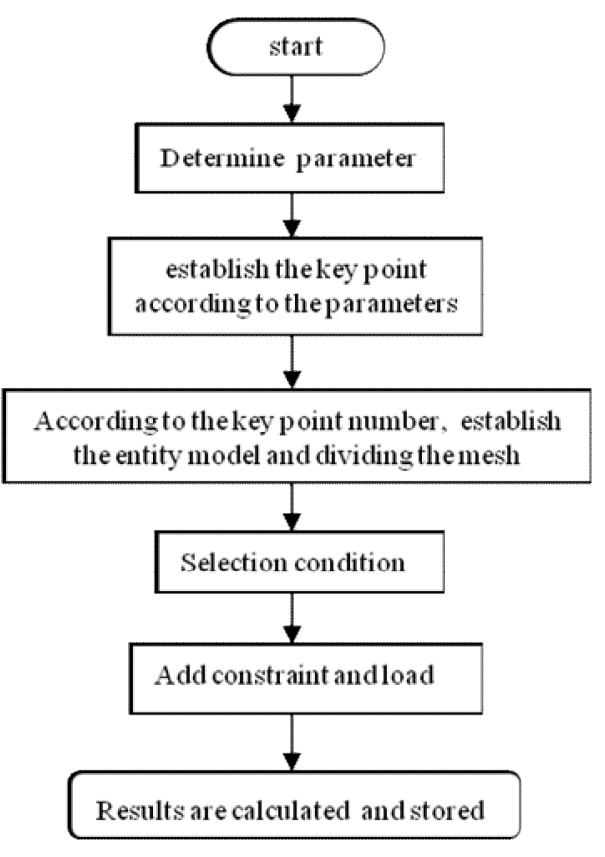

Fig. 2 with friendly interface, simple operation, practical function for the principle, it can be able to achieve the same form of different types of gantry crane for finite element analysis, and easy to observe and analyze the results. Because the Visual Studio call to ANSYS by always running in the background, the ANSYS interface does not appear in the process of calculation, therefore, for never used the design of ANSYS can easily use this system to analyze the structure of the finite element, and an intuitive way to look at the results to evaluate the rationality of the structure. According to the specific situation, the system selects the following 2 conditions to analyze:

(1) The trolley is in the middle of the span, load lifting, considering the effect of dynamic load, wind load;

(2) The trolley is loaded with effective cantilever, load lifting, considering the effect of dynamic load, wind load.

The strength and stiffness of the structure can be analyzed by these two kinds of working conditions. The realization of the system function is divided into the following steps:

(1) Entry system

"General purpose U type gantry crane structure design system - finite element simulation analysis" of the interface as shown in Fig. 3.

(2) Input parameter

(3) Generates the command stream file and calls the ANSYS calculation module to get the result.

(4) Read and display the calculation results 


\section{The development and application of parameterized modeling program for gantry crane}

\section{Modeling and analysis}

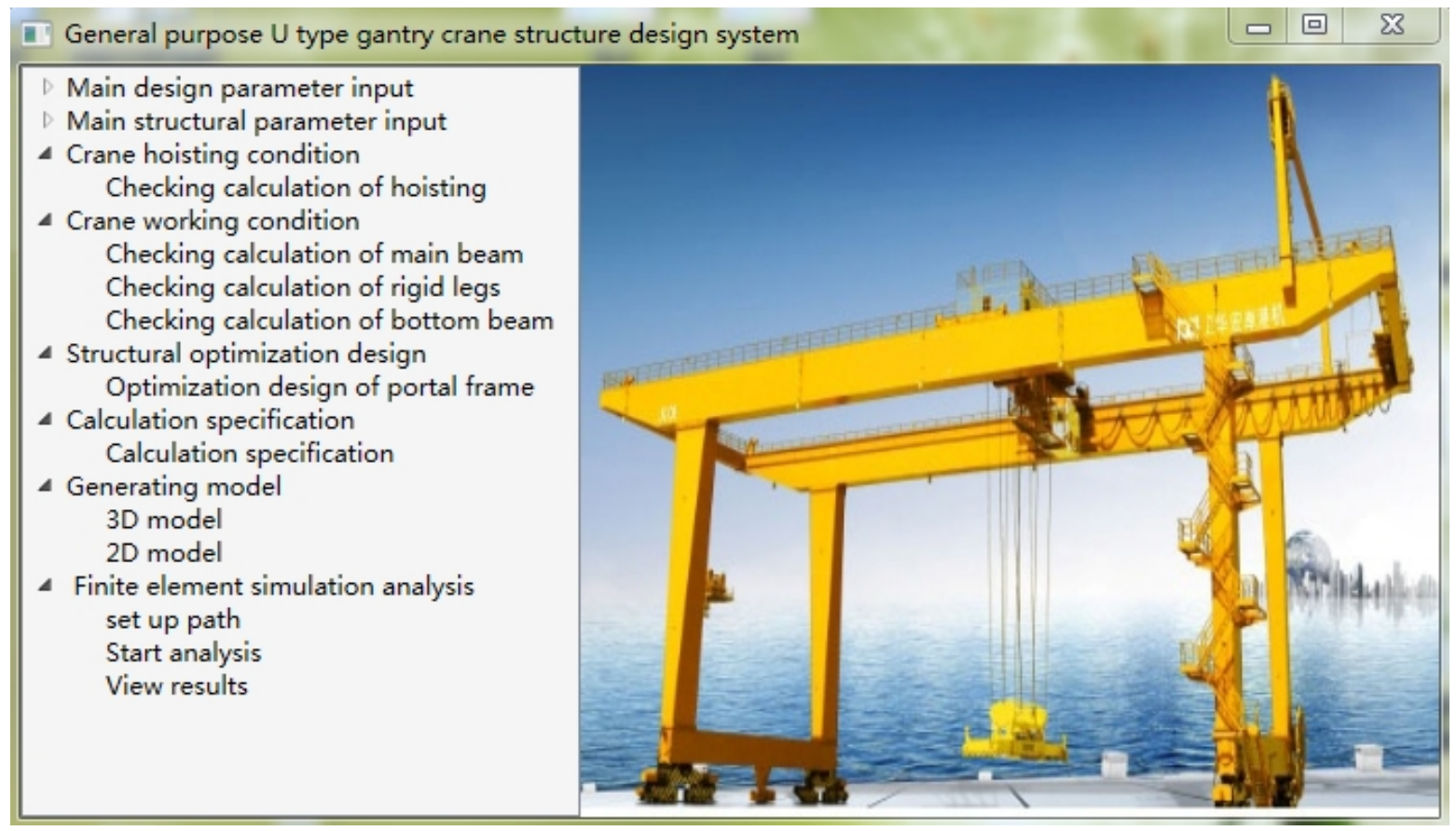

Fig. 3

$\mathrm{U}$ type gantry crane as an example of $150 \mathrm{t}-28 \mathrm{~m}$, using the parametric modeling program is developed based on the above principle, the rapid establishment of finite element model, through the definition of element types, real constants, material properties, meshing, loading ,constraint and a series of operations, can be solved and analyzed. The whole finite element model is established with the analysis of the part of the code as follows:

ESIZE, WGCC

AMESH, ALL

VATT, 1, 1, 2, 0

VMESH, ALL

/SOLU

DA, 319, UX\$\$DA, 319, UZ\$\$DA, 319, ROTX\$DA, 319, ROTZ

DA, 320, UX\$\$DA, 320, UY\$\$DA, 320, UZ\$\$DA, 320, ROTX\$\$DA, 320, ROTZ

DA, 321, UX\$\$DA, 321, UZ\$\$DA, 321, ROTX\$DA, 321, ROTZ

DA, 322, UX\$\$DA, 322, UY\$\$DA, 322, UZ\$\$DA, 322, ROTX\$\$DA, 322, ROTZ

ACEL, 0, , 9.8! Add inertia load and gravity load

ASEL, S, LOC, Y, -GD_XC/2-ZL_B/1000

ASEL, A, LOC, Y, GD_XC/2

ASEL, A, , , 325, 329, 4

ASEL, A, , , 682, 689, 7

ASEL, A, , , 690, 697, 7

ASEL, A, , , 731, 733, 1

ASEL, A, , , 743, 745, 1

SFA, ALL, 1, PRES, $1.8 * 1.5 * 250$

ALLSEL, ALL 


\section{SOLVE}

According to the actual operation characteristics and operation conditions of the crane, under different working conditions, considering the probability of various loads appear in the actual work, according to the structure of the most unfavorable situation, reasonable combination of various loads which may occur. Fig. 1 to Fig. 4 respectively is the crane two kinds of working conditions to discuss and analysis:

Condition 1: the trolley is located in the main beam mid-span the full load from the lifting of the working conditions, that is, the car runs to the main beam in the middle of the rest, and the full load of lifting goods. After the analysis, Displacement and the stress nephogram are got and shown in Fig. 4 and Fig. 5:

Fig. 4 equivalent main beam stress nephogram of the maximum equivalent stress of the main beam is $66.8 \mathrm{MPa}$, Fig. 5 the displacement distribution of the main beam can be known, the maximum deformation at mid span position, the maximum deflection is $19.833 \mathrm{~mm}$, based on the theoretical results: the fully loaded trolley is in the middle of the span, the maximum stress is 67.81MPa. The maximum deformation $19.6 \mathrm{~mm}$. The results of finite element analysis are compared with the theoretical calculation results, the maximum stress difference is about $1.48 \%$, and the maximum deformation difference is about $1.2 \%$.

Condition 2: the trolley is located in the main beam across the end of full load from the lifting of the working conditions, that is, when the car runs to the main beam left limit or the right limit, fully loaded to lift the goods.

The maximum equivalent stress of the main beam is $87.2 \mathrm{MPa}$, which occurs at the side of the supporting leg, when the trolley is fully loaded at the end-span from the Fig. 6. The displacement of the main beam from Fig. 7 can be known, trolley is located the end-span of the limit position the maximum deformation is $20.041 \mathrm{~mm}$, based on the theoretical results: the fully loaded trolley at the cantilever end, the end-span of the maximum stress is $91.505 \mathrm{MPa}$, the maximum deformation of the cantilever $21.33 \mathrm{~mm}$. The results of finite element analysis are compared with the theoretical calculation results, the maximum stress difference is about $4.7 \%$, and the maximum deformation difference is about $6 \%$ at the end of the cantilever.

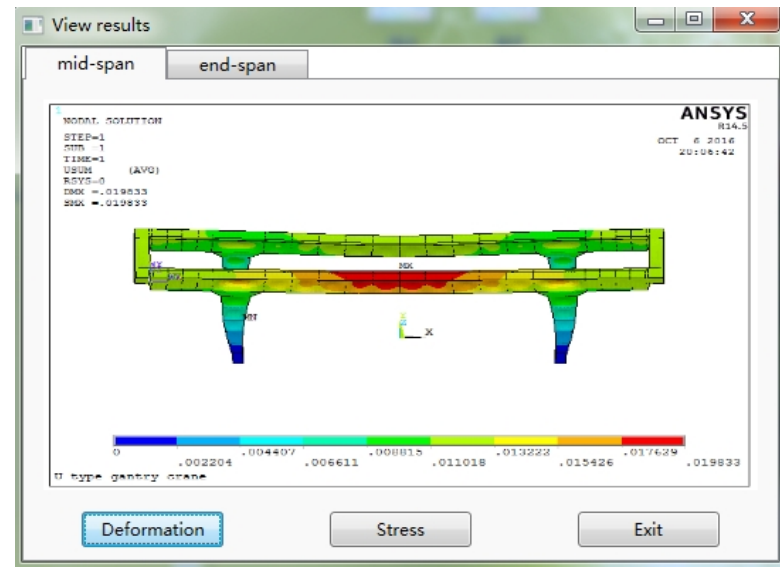

Fig. 4 Deformation cloud in mid-span

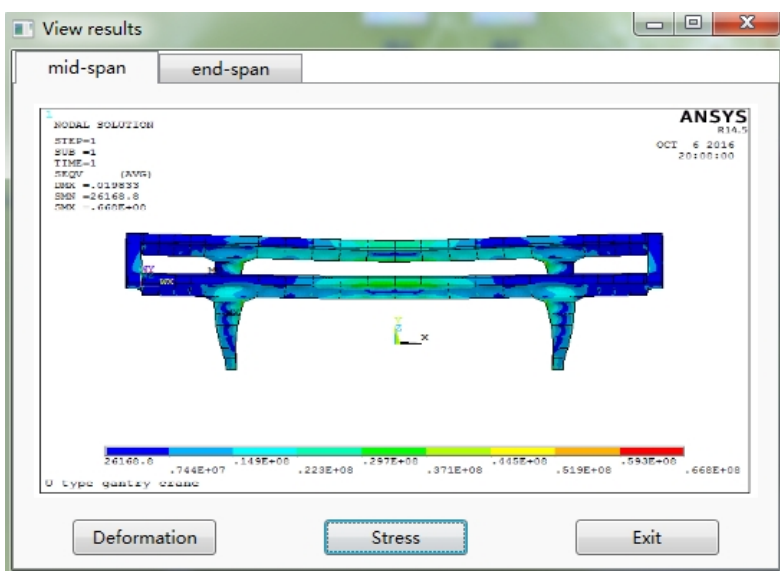

Fig. 5 Stress cloud in mid-span 


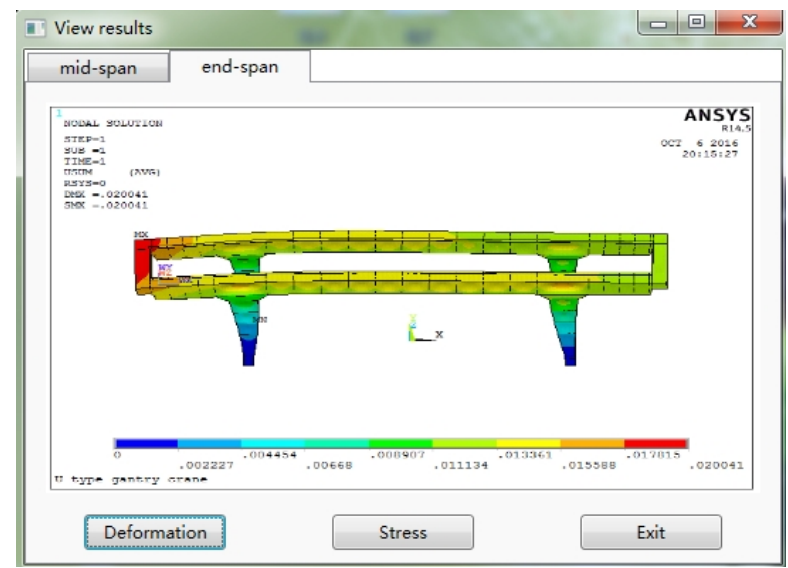

Fig. 6 Deformation cloud in end-span

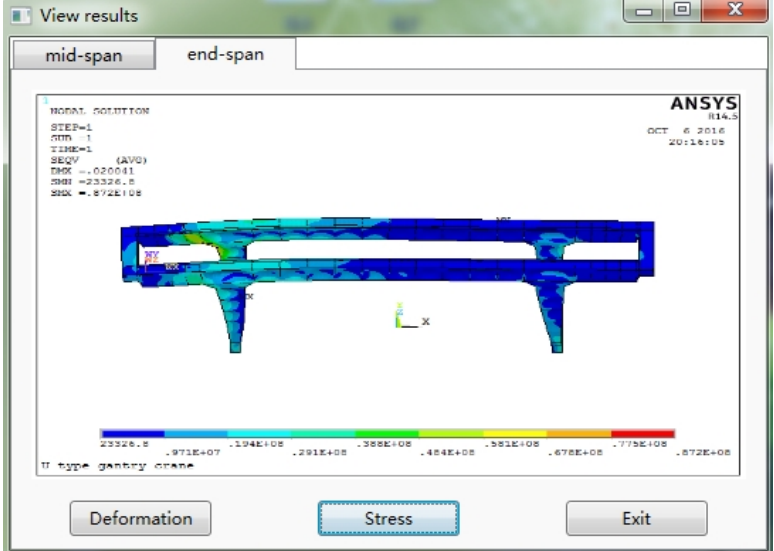

Fig. 7 Stress cloud in end-span

From the analysis of the above two conditions, we can see that the finite element model, the constraint and the load are in accordance with the theoretical calculation results, which meet the design requirements.

\section{Convergence analysis}

The ANSYS finite element analysis result is consistent with the theoretical calculation result, but it is not enough to prove the correctness of the finite element model. Because of the model in this paper, the welding and flange, convex platform are simplified in the modeling process, using concentrated load instead of the wheel and the rail contact surface, This will make the role of the board and the board connected directly and concentrated load will form a sharp angle. Based on the elastic theory, in the corner there will be stress concentration, the stress value is infinite, the finite element model for elastic theory caused by the difference of the load and the structure of the model stress calculation result of the infinite is called singular stress ${ }^{[7]}$.

In order to avoid the appearance of stress singularity, the mesh size of the model and the connection between the plate and the plate are treated:

(1) When establishing the crane trolley track model, force a concentrated load on the track, and ignore the influence of track when the finite element analysis results are post-processed. Only considering the stress and deformation of the main beam of the crane;

(2) The connection between the main beam and the supporting legs of the crane is processed by the circular arc, the formation of excessive fillet, avoid corner joints;

(3) Control mesh density, The connection between the main beam and the supporting legs of the crane as well as the main beam own part adopts relatively sparse.

Take the fully loaded trolley is located end-span of the crane main beam under the most dangerous condition in several different mesh size for convergence analysis results verified that the model, table 1 shows the maximum equivalent stress under different mesh size, Fig. 8 to Fig. 11 show that the results of finite element stress analysis under different mesh densities.

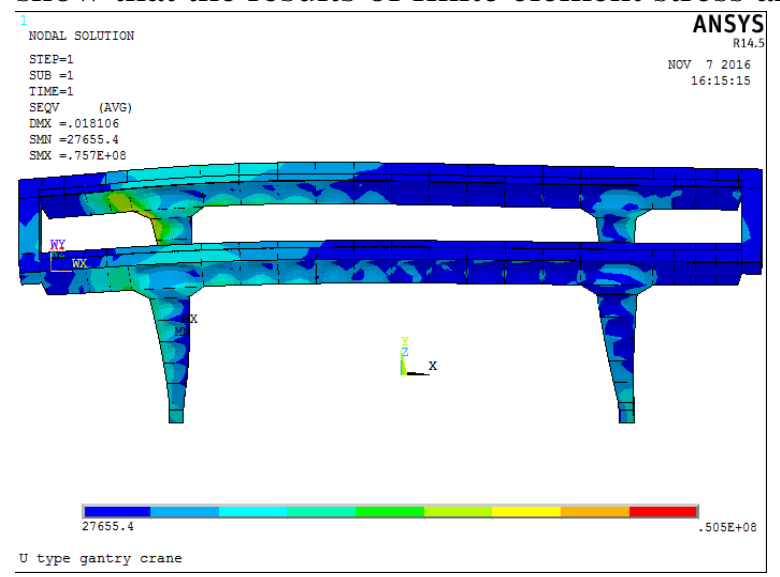

Fig8 Grid size $0.5 \mathrm{~m}$

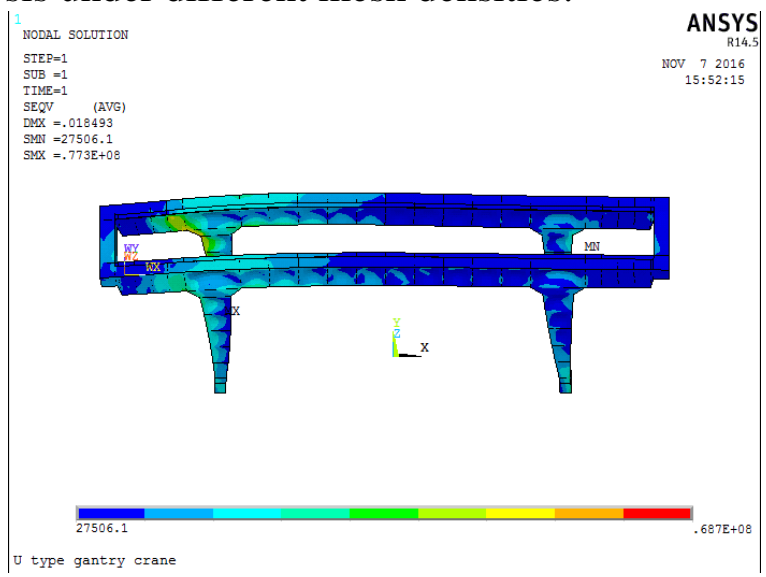

Fig9 Grid size $0.4 \mathrm{~m}$ 


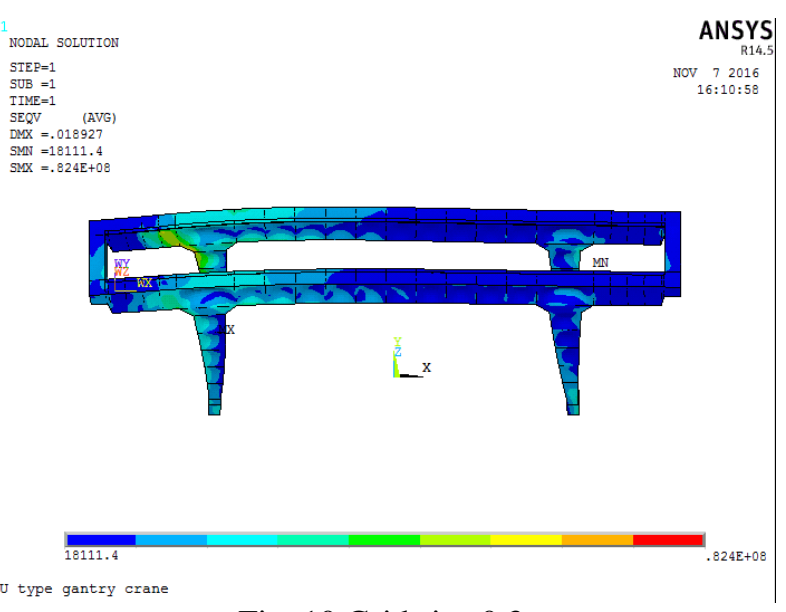

Fig. 10 Grid size $0.3 \mathrm{~m}$

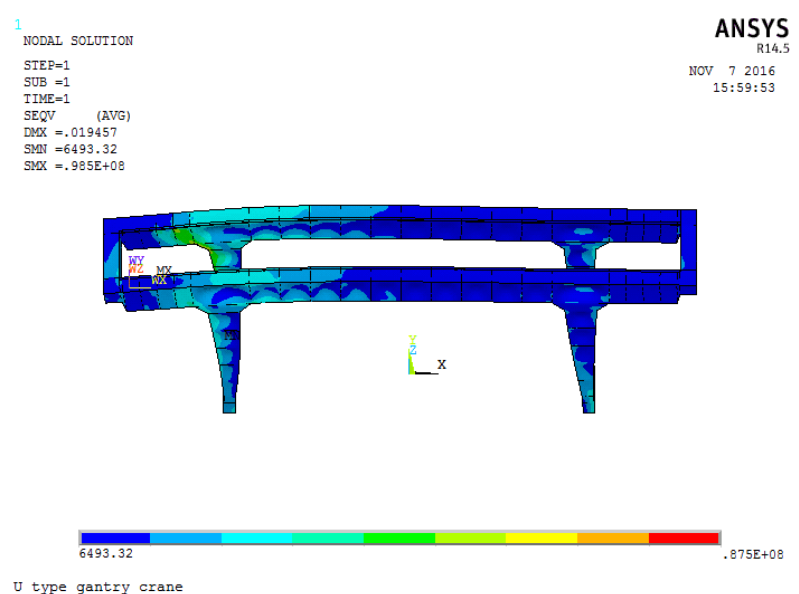

Fig. 11 Grid size $0.2 \mathrm{~m}$

From table 1 and Fig. 8 to Fig. 11 shows that: as the mesh size decreases by the mesh density from sparse to dense, even if the maximum equivalent stress value in gradually become larger, but the percentage of the equivalent stress increment and the relative error percentage of the maximum equivalent stress between adjacent mesh dimensions is smaller and smaller, it can be concluded that as the mesh size tends to infinity, the maximum equivalent stress will converge to a certain value, which can be explained that the finite element analysis result of the model is convergent, and the model is reasonable and the finite element analysis results are reliable.

Table 1 The maximum equivalent stress under the different grid density

\begin{tabular}{|c|c|c|c|c|}
\hline Mesh size $(\mathrm{m})$ & 0.5 & 0.4 & 0.3 & 0.2 \\
\hline Maximum equivalent stress(MPa) & 50.5 & 68.7 & 82.4 & 87.5 \\
\hline Equivalent stress increment(MPa) & - & 18.2 & 13.7 & 5.1 \\
\hline Relative error(\%) & - & 36 & 19.9 & 6.2 \\
\hline
\end{tabular}

\section{Conclusion}

In this paper, the combination of C\# and APDL developed a finite element analysis system of a gantry crane parameters, the system has the advantages of simple operation, through analysis the correlation of the overall structure size of the gantry crane, considering the structure characteristics of different types of gantry crane, realize the parametric representation of the overall structure size, while the use of APDL language and macro technology. It realizes the parametric modeling and simulation analysis model for gantry crane, Through the example of gantry crane parametric modeling and simulation analysis show that the method presented in this paper is simple and practical, which provides an effective means for the rapid finite element analysis and application of different types of gantry crane. At the same time, the design of the software system to improve the design efficiency of the gantry crane, shorten the development cycle of the product, it has great significance to the development and design of the following crane.

\section{Reference}

[1] Gening $\mathrm{Xu}$, Yuanfang Tao, Liangbao Wei. 3D parametric modeling and analysis of finite element method based on APDL language. Taiyuan Heavy Machinery Institute, 2013.

[2] Fuchi Wang, Zhaohui Zhang. ANSYS10.0 finite element analysis theory and engineering application [M]. Beijing Industry Press, 2006.

[3] Peng Wu, Hong Zeng, Mai Han. The realization method of the secondary development technology based on ANSYS[J]. Journal of Liaoning University of Technology: Natural Science Edition, 2004. 
[4] Chunqiang Hao. C\# foundation and an example of the tutorial [M]. Beijing: China Electric Power Press, 2005.

[5] Game studio. APDL parametric finite element analysis technology and its application examples [M]. Beijing: China Water Conservancy and Hydropower Press,2004.

[6] Ning Zhou. APDL advanced engineering application examples and secondary development. Beijing: China Water Conservancy and Hydropower Press,2007.

[7] Qisong Qi. Parametric design and finite element analysis system of U type gantry crane[D]. Taiyuan University of Science and Technology, 2013. 